\title{
Usability of Nomadic User Interfaces
}

\author{
Walter Dees \\ Philips Research Laboratories, High Tech Campus 34, Eindhoven, The Netherlands \\ walter.dees@philips.com
}

\begin{abstract}
During the last decade, a number of research activities have been performed to enable user interfaces and the underlying user activities to be migrated from one device to another. We call this "Nomadic User Interfaces". The primary goal of these research activities has been to develop the technologies to enable this. However, not much is known about the usability aspects of Nomadic User Interfaces. In this paper we present the results of three different user tests that we conducted to investigate the usefulness and the usability issues of several prototype Nomadic User Interface systems that we developed.
\end{abstract}

Keywords: Nomadic User Interfaces, UI Adaptation, Migratory Interfaces, Pervasive Applications, Ubiquitous Access, Device Independence, Multi-device UI Authoring, UI Consistency, Session/Activity Migration, Session Mobility, Multimodal Interfaces, Remote User Interfaces, User Study, Usability Guidelines.

\section{Introduction}

Interconnectivity of devices using technologies such as Bluetooth, DLNA, and broadband allows new usage scenarios. One of the opportunities enabled by the enhanced interconnectivity offered by these technologies is to give people more flexibility in when and where to access their favorite content and applications. The next step in enhancing these types of scenarios is through a concept that we call "Nomadic User Interfaces" (also known as "Migratory User Interfaces" [1]). The main idea behind this concept is that interactive user interfaces offered by an application in the network can be freely migrated from any stationary and portable device inside the network to other devices in the network. It allows users to start an activity on one device and continue this activity on another device. This is illustrated in Figure 1.

Examples of Nomadic User Interface scenarios may include a user creating a playlist on a tablet device, and then start playing the contents of this playlist on the living room TV and/or stereo system. When the user goes to bed, he can continue to finish the playlist in the bedroom, skip a few items and stop the playlist through a user interface on the bedroom TV. Another example could be a user typing a message on a mobile phone and continue typing the message on a big screen TV with a wireless keyboard.

The topics underlying Nomadic User Interfaces, such as UI adaptation techniques, model based design of user interfaces, (semi-)automatic generation of multi-device user interfaces, session migration techniques and remote user interface technologies 
have been a topic of research for several years. Examples of research results in this area include: TeresaXML/Migration project [2], UsiXML/MigriXML [3], UI Plasticity [4], Supple [5], and Multi-level Stylesheets [6][7].

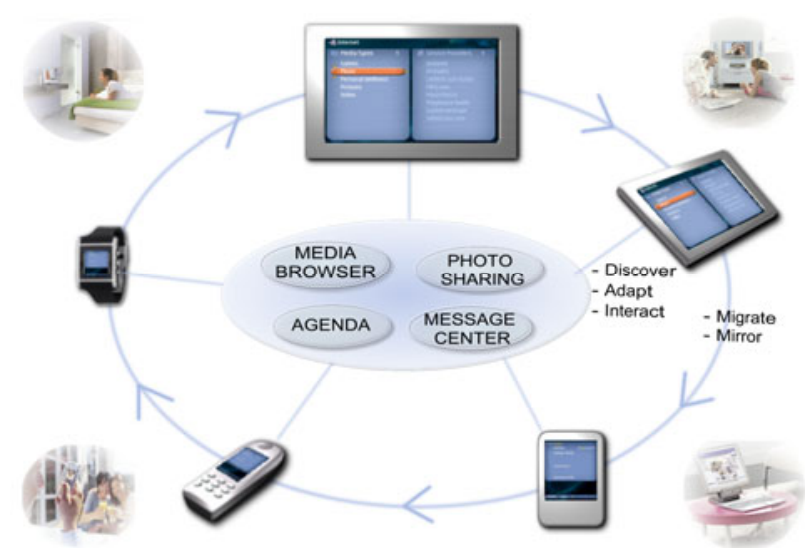

Fig. 1. Nomadic User Interfaces

The primary goal of most of these research activities has been to get the technologies in place to enable concepts such as Nomadic User Interfaces. Not much research has been done to measure the usability criteria of Nomadic User Interfaces and to identify the usability issues of such a system in practice. Therefore, we have implemented several prototypes, and conducted three different user tests to investigate this.

\section{Study 1: Exploratory Study}

\subsection{Introduction}

The first user study was a qualitative study on the usefulness and usability issues of Nomadic User Interfaces, with a focus on in-home usage. The prototype created for this study allows migration of the GUI of a media browser application to an HD and SD resolution television, mobile phone, PDA, tablet PC and an iPronto remote control. The prototype supported both push- and pull-migration. This means that a user can instruct the system to move (i.e. "push") the current activity to another device, or at any moment use another device to discover a list of currently active and suspended activities, and continue (i.e. "pull") the activity to the device that the user is currently using.

\subsection{Setup of the Experiment}

The experiment was conducted at the Philips ExperienceLab [8], and involved eight sessions of approximately two-hours in length. The participants of the experiment were 8 couples (mostly married or couples living together). Their familiarity to networking concepts ranged from high understanding of the underlying technical mechanisms to 
no prior experience of using networked systems, while there was a bias towards educated and financially fortunate people. However, this bias was not considered as misleading since such users seem to be the initial target group of the Nomadic User Interface concept.

After a short introduction of the experiment and the system, the users were given complete freedom to transfer their activities from one device to another, to different devices located throughout the home, i.e. a living room TV, a bedroom TV, a touch screen display in the kitchen, a tablet, a mobile phone and a PDA. During the exploration phase the experimenter was present, observing the users at a distance, only interrupting the participants when absolutely needed. Subsequently, certain role playing scenarios were employed, such as watching pictures that were stored on a mobile phone together on a TV, creating a playlist, and moving a session from the TV downstairs to a TV upstairs. The participants were asked to "think aloud", in order to observe their thoughts and behaviors. The entire two-hour session was recorded using the camera infrastructure of the Philips ExperienceLab, for further analysis.

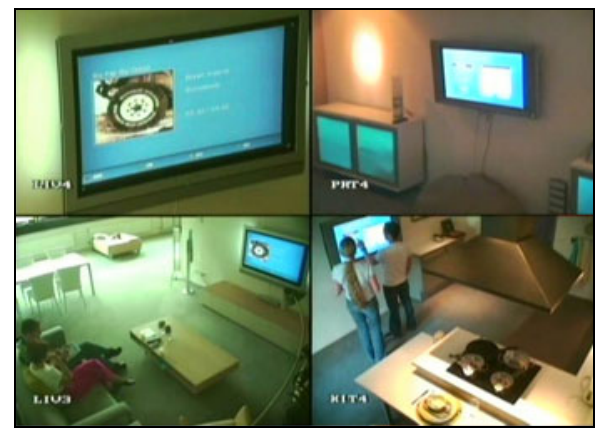

Fig. 2. Views from the experiment

\subsection{Analysis of the Results}

As mentioned, the prototype supported both push- and pull-migration. The pullmigration was considered to be the most favorite of the two. The reasons that were given for this are that the push-migration paradigm entails premature commitment while in-home interactions are rarely planned. It also is unclear at the moment they push the activity to another device in another room, whether or not that device is actually available (e.g. switched on, not occupied by someone else). Furthermore, pushmigration does not deal with the issue of the "dead time" during the relocation. Users mentioned for example that they may want to transfer their current activity to the bedroom TV, but first want to go and brush their teeth. People want to be sure that the status of their session will be kept intact. Although the prototype supported automatic suspension of the activities, this does not give sufficient feel of control. It was felt that it was removing control from them, and that they rather have an explicit action to suspend the UI (before migration) than automatic suspension by the system. The "suspend" or "pause" metaphor is very familiar to the users and therefore should be considered for this type of scenarios. Also, during the study people expressed a need for 
having the ability to mirror and split the UI across multiple devices, next to pull and push migration.

One of the main issues that this study revealed is that the notion of networked activities is not readily comprehensible by all users. They were confused as to where the session was located, and felt a need to have some indication with which device they are communicating and where the data comes from. During the experiment we also noticed a hesitant attitude towards the usefulness of migrating activities that involve a high degree of interactivity. This may be due to the perception that users have about each of the individual devices, and the capabilities/limitations of interacting with some of these devices. This requires further study.

Based on the feedback from the experiment, we were able to classify some of the different user needs relevant to the concept of Nomadic User Interfaces. Figure 3 shows a possible classification of user needs for Nomadic User Interfaces in domestic environments. Next to the mobility of activities across devices, triggered by certain interruptions, it also shows the relevance of Nomadic User Interfaces in cases where an activity is started on one device, but a device which offers enhanced interaction is available in the vicinity, e.g. moving the activity from a mobile phone to a large screen TV.

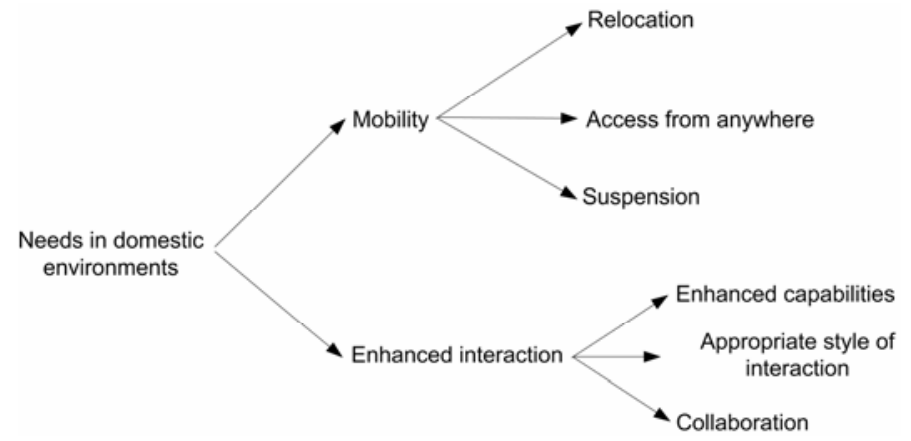

Fig. 3. Classification of user needs relevant to Nomadic User Interfaces

\section{Study 2: Deeper Analysis}

\subsection{Introduction}

The main focus of the second user study was to gain a deeper insight in the user needs and usability requirements regarding Nomadic User Interfaces, and in particular different forms of migration and session state recovery. In order to test this, a new prototype was implemented that supports the following types of migration:

- The first is total migration, whereby a running activity can be moved from one device to another in its entirety. This can be done using either a push mechanism or pull mechanism, as described in the previous section. Once the current activity is transferred to the new device, the device from which the activity was moved 
becomes passive and the new device becomes active. The user starts interacting with the new device using available interaction means of the new device.

- The second is partial migration and in this case a running activity is transferred partially from one device to another. This distributes or splits the user interface over two devices. For example, once the user discovers a large screen TV in the vicinity, the user moves the activity that is running on the mobile phone to the TV, whereby the control part remains on the mobile device and the presentation part is moved to the TV screen. An example of this is given in Figure 8.

- The third is mirroring (also known as cloning), whereby the UI is shown on both devices simultaneously and both UIs are kept in sync.

Furthermore, for both partial and total migration different types of state recovery granularity, such as session level, task level, and action level granularity (as defined in [9]) were supported by the prototype. The main target devices of this second prototype were a mobile phone, a TV and a PC. A photo manager/sharing application, with which the users could sort their photo collection, annotate pictures, create albums and slideshows, and share photos with friends, was chosen as the carrier application.

\subsection{Setup of the Experiment}

In total 5 users, all well-educated people, participated in sessions of 1.5 hours each. The study was a controlled user study conducted at the Philips ExperienceLab whereby users had to follow different tasks in a task book. These tasks were steps inside a larger scenario that included various forms of migration due to different kinds of interruptions (e.g. receiving a message from a friend whilst organizing pictures). Some tasks had to be repeated several times in order to get some insight into the preferred state recovery granularity.

The user had to fill in a questionnaire each time a task was completed. There was also a pre- and post-test questionnaire which included more general questions about session migration and its usefulness.

\subsection{Analysis of the Results}

The following figure shows the result of some of the questions in the questionnaires that were filled out.

These results show that the participants appreciated the session migration feature, and found it useful to have such feature. We also asked the participants some questions about which activities they currently perform on more than one device and on more than one location. In total, the participants suggested 11 different activities with which they currently have or would like to have a continuous involvement with across devices and locations, i.e. activities for which a session migration feature could be very useful. Chatting and gaming were amongst the most frequently suggested.

Regarding the question about whether people prefer total migration, partial migration or mirroring when moving an activity from mobile phone to $\mathrm{TV}$, people ranked them as follows: partial migration was clearly preferred by 3 users, cloning was preferred by 1 user, and push migration by 1 user. Unfortunately, the number of participants for this experiment was very low, which makes these results inconclusive. 
The test revealed that we should always aim to recover as much of the UI state information as possible to ease the interaction continuity. The effect does however become less the longer it takes between a session save and a session restore.

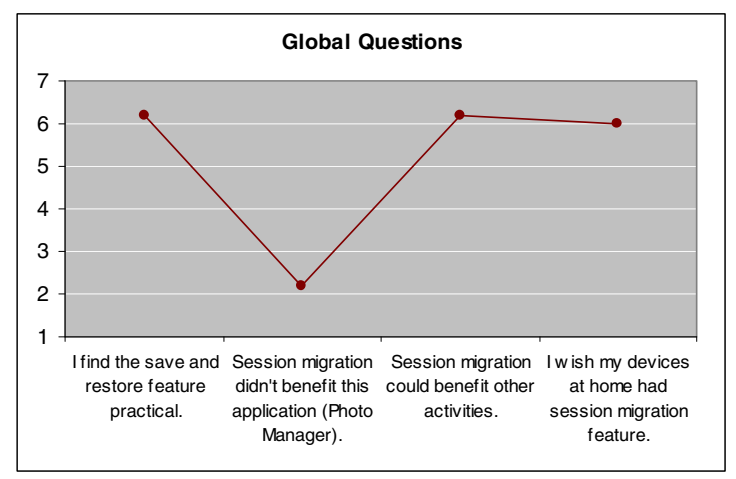

Fig. 4. Results of global questions questionnaire of second user test using 7-point Likert scale

\section{Study 3: Consistency of Multi-device Interfaces}

\subsection{Introduction}

The main focus of the third study was to investigate the consistency requirements of multi-device user interfaces in a controlled manner. Research questions included: Which consistency parameters are most relevant when moving a session from one device to another for the user to not get lost. Do people prefer intra-device or intraapplication consistency? Can we define some consistency and multi-device UI authoring guidelines for Nomadic User Interface systems?

For investigating these questions, the second prototype was altered a bit and a number of variations in the interface and interaction (both consistent and inconsistent) were introduced into the system. There were some intra-device inconsistencies and inter-device inconsistencies. Globally, six different types of interface and interaction variations were implemented for two different devices: mobile phone and TV. These interface/interaction variations included differences in look\&feel parameters, layout,
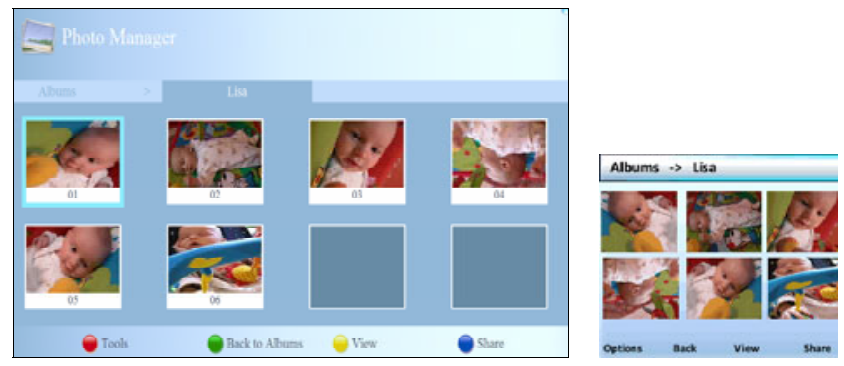

Fig. 5. Example of two corresponding user interfaces 
widget type, and touch/key-based interaction. Figure 5 shows an example of two corresponding user interfaces with a consistent colour scheme, but with different interaction style.

\subsection{Setup of the Experiment}

In total, 24 users participated in this user study. A proper participant screening was done before confirming participants for the study. We tried to have both expert users (who are familiar with some of the technologies and devices used) and novice users (who are not familiar with the technologies and devices used). The research model in Figure 6 shows which attributes were controlled and which were manipulated during the experiment.

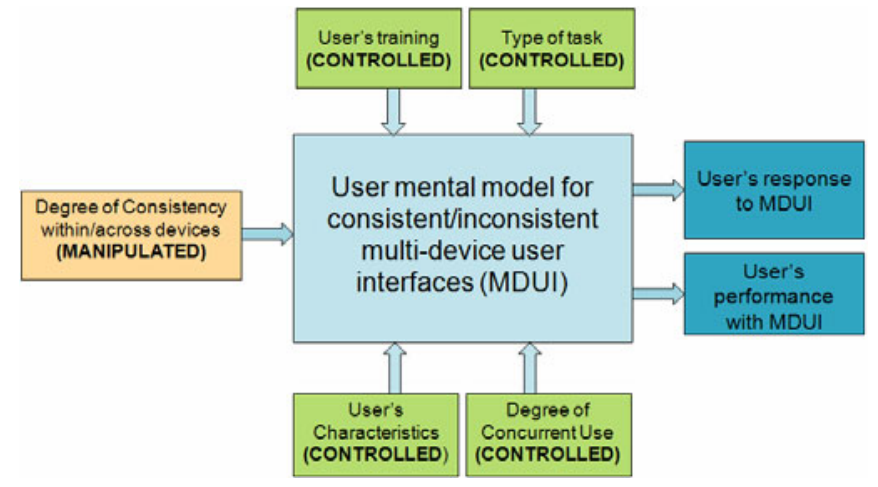

Fig. 6. Research model used for experiment

\subsection{Analysis of the Results}

The results of the usability study gave a good insight into the user's mental model that they constructed when they moved between two consistent user interfaces and inconsistent user interfaces. It also revealed which consistency parameters are more important than others. The results reveal that one of the most troublesome inconsistencies is interaction inconsistency (e.g. from remote control interface to touch interface). The impact of color inconsistency was also high. The use of consistent colors across interfaces running on two different devices gives a feel of connectedness and users perceive such an application running on two different devices as a single application.

The results revealed that consistency is an important attribute for enhancing the overall user experience (see Figure 7).

However, it is also learned that consistency is not the only requirement to ensure cross-device usability and in some cases it is important to sacrifice consistency for getting the benefits of local platform capabilities. For example partial migration, whereby the user interface output is rendered on the TV whilst the user input is done on the mobile phone, is very inconsistent (see Figure 7), but has low cognitive overhead, and was perceived as a very natural way of interacting. Also, during some of the tasks, users explicitly chose and preferred using touch screen based interaction instead 


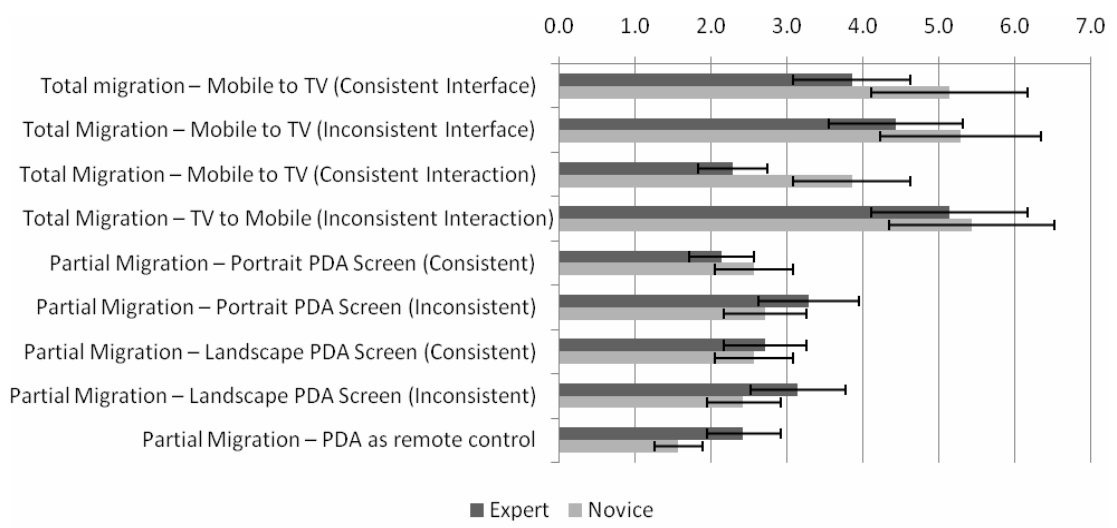

Fig. 7. Cognitive overhead of session migration (lower score means less overhead and therefore easier to use)
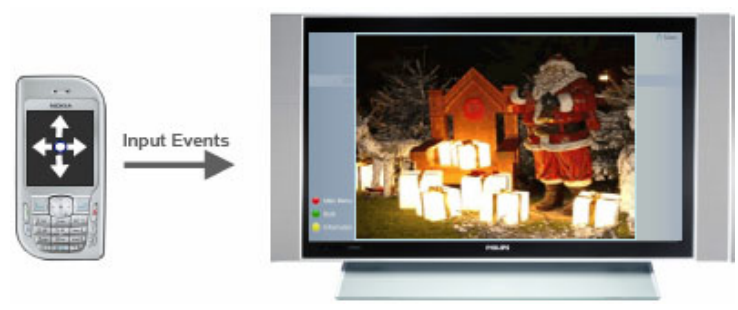

Fig. 8. Partial migration

of keypad based interaction although keypad-based interaction was more consistent to the remote control based interaction used for TV.

Based on the results of this usability study, several multi-device UI authoring guidelines were formulated. These guidelines can assist designers in transforming one platform specific interface into another and to generally improve the UI authoring process for multi-device user interfaces. Examples of such guidelines include:

- Wherever possible, design for cross-platform consistency instead of local (i.e. native) look and feel consistency, in particular maintain cross-device consistency of color.

- Make sure that the "point of focus" of the user is kept consistent across migrations.

- Scale items instead of repositioning or re-layouting the items (e.g. by adding whitespaces) when moving to a different screen size.

- Partial migration requires a carefully designed UI that is consistent with the interaction style and menu style of the input device, but also consistent with screen layout of the output device.

- Instead of renaming long labels of a widget, apply truncation rules (e.g. replace "accept changes" with "accept..." or "accept chan...") when moving to a smaller screen size. For normal text, it is better to define a set of text transformation rules where long text can be replaced with semantically equivalent short text. 
- Whilst replacing a widget which has more than one item with another widget, do not change the order of items or item selection colour.

We also analyzed the consistency impact of various visual element manipulations. Figure 9 shows which differences between two user interfaces are considered more harmful than others, when moving from one platform to another. The changes on the top are less harmful and are thus perceived as less inconsistent. Changes at the bottom are more harmful and are perceived as inconsistent.
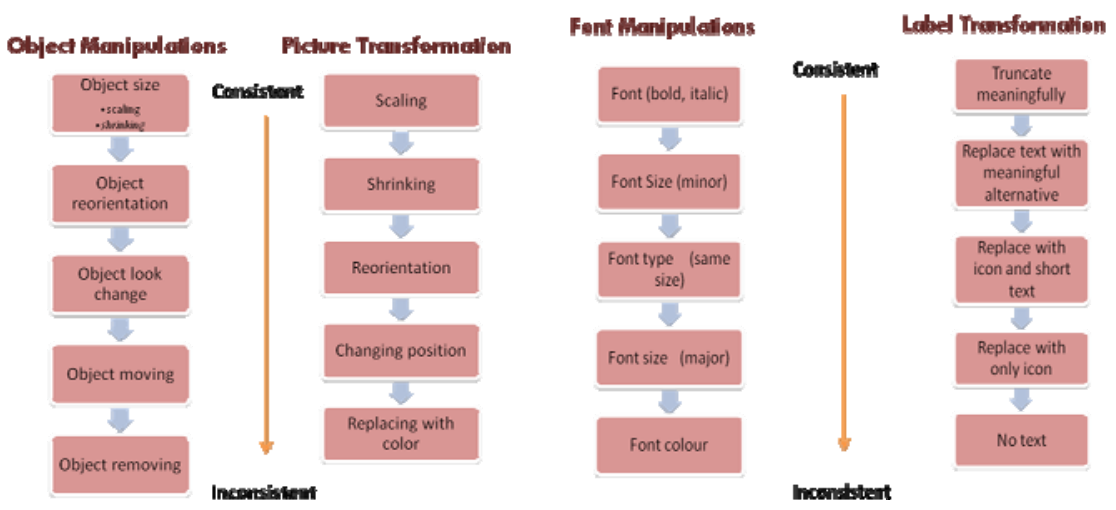

Fig. 9. The impact of different manipulations on the perceived consistency

\section{Conclusions}

The three studies as presented in this paper provide insight in some of the user interface needs and issues behind the concept of Nomadic User Interfaces.

The first user study showed that people like the pull-migration paradigm over the push-migration paradigm. Furthermore, the study revealed a need for users to have explicit control over suspending/pausing the user interface, rather than the system doing this automatically. The study also allowed us to get better insights in the user needs of Nomadic User Interfaces (such as mobility and enhanced user interaction offered by another device), and some of the practical problems and issues.

During the second study we gained more insights into which type of migration (i.e. total migration, partial migration or mirroring) and which type of state recovery granularity people prefer when moving an activity from one device to another. However, due to the low number of participants the results of this test were rather inconclusive, although the study did confirm our expectation that we should always aim to recover as much of the UI state information as possible to ease the interaction continuity.

The third study revealed the requirements related to the consistency of user interfaces across devices, when used in a Nomadic User Interface context. It showed that consistency is a very important factor in usability of multi-device user interfaces. However, the results also show that it is not the only factor. Based on this study, we formulated a number of design guidelines that will help to improve the UI authoring 
process for user interfaces that span multiple devices or that can move freely from one device to another.

Further studies are needed to get a better understanding of the impact of the capabilities/limitations of certain devices on the willingness to migrate certain activities from one device to another. Also several next steps would have to be made in improving the Nomadic User Interface framework, its user interaction concepts, and also the technologies and tools to create Nomadic User Interfaces, in order to see if Nomadic User Interfaces really has the potential to become successful.

The results of these user tests, including the problems and issues that have been identified during these studies, are therefore valuable input for further research and future deployments in this area.

Acknowledgements. This research could not have been done without the help and valuable support of P. Shrubsole and R. Haakma from Philips Research, Prof. Dr. P.M.E. de Bra, Dr. E. Karapanos, and B. Golsteijn from Eindhoven University of Technology, S. Shahid from Tilburg University, Prof. Dr. L. Hardman from CWI Amsterdam, N. Frame from the University of Bordeaux, and J. Coutaz and G. Calvary from the University of Grenoble.

\section{References}

1. Berti, S., Paternó, F., Santoro, C.: A Taxonomy for Migratory User Interfaces. In: Gilroy, S.W., Harrison, M.D. (eds.) DSV-IS 2005. LNCS, vol. 3941, pp. 149-160. Springer, Heidelberg (2006)

2. Migratory User Interfaces, http://giove.isti.cnr.it/tools/Migration/

3. UsiXML - USer Interface eXtensible Markup Language, http : / /www . usixml . org/

4. Coutaz, J.: User interface plasticity: model driven engineering to the limit! In: Proceedings of the 2nd ACM SIGCHI Symposium on Engineering Interactive Computing Systems, EICS 2010, pp. 1-8. ACM, New York (2010)

5. Gajos, K., Weld, D., Wobbrock, J.: Automatically generating personalized user interfaces with SUPPLE. J. Artificial Intelligence 174, 910-950 (2010)

6. Dees, W.: Handling device diversity through multi-level stylesheets. In: Proceedings of the 9th International Conference on Intelligent User Interfaces, IUI 2004, pp. 229-231. ACM, New York (2004)

7. Dees, W.: Device Independent Layout and Style Editing Using Multi-Level Style Sheets. In: Proceedings of the Sixth International Conference on Computer-Aided Design of User Interfaces V, pp. 183-190. Springer, Heidelberg (2007)

8. Philips Research - ExperienceLab, http://www.research.philips.com/focused/experiencelab.html

9. Balme, L., Alvaro, X., Calvary, G., Demeure, A., Sottet, J.-S.: An MDE-SOA Approach to Support Plastic User Interfaces in Ambient Spaces. In: Stephanidis, C. (ed.) UAHCI 2007 (Part II). LNCS, vol. 4555, pp. 63-72. Springer, Heidelberg (2007) 